To appear in the International Journal of Computer Mathematics

Vol. 00, No. 00, September 2013, 1-20

\title{
Insight into Spontaneous Recurrent Calcium Waves in a 3-D Cardiac Cell Based on Analysis of a 1-D Deterministic Model
}

\author{
Zana Coulibaly, Matthias K. Gobbert, and Bradford E. Peercy* \\ Department of Mathematics and Statistics, University of Maryland, Baltimore County \\ (13 September 2013)
}

\begin{abstract}
Calcium dynamics in a cardiac cell are described by a system of 3-D non-linear stochastic partial differential equations. To obtain solutions that have biophysical properties, it is necessary to explore the model parameter space. To decrease the complexity of the parameter search, we reduce the 3-D stochastic model to a 1-D deterministic model. The reduction of the problem from 3-D to 1-D is done through an asymptotic approximation after non-dimensionalization and based on rational biophysical assumptions of the 3-D domain; the stochastic to deterministic transformation is based on the regular property of the 3-D solution. The result of the model reduction proves very effective in reducing the time required to get qualitative as well as quantitative information about parameter regions in the 3-D stochastic model including calcium dynamics (sparks, wave propagation, and recovery) observed in cardiac cells.
\end{abstract}

Keywords: calcium wave; cardiac cell; asymptotic approximation; 3-D stochastic simulation; parallel computation

AMS Subject Classification: 35K57, 35B40, 65M60, 65Y05, 92C45

\section{Introduction}

Various computational methods and computational architectures have been used to model the diffusion of calcium in cardiac cells (see [5] for modeling through ODEs; see [10] for modeling through stochastic PDEs modeling; and see [13] for Monte Carlo simulations using a GPU). Although challenging to compute over application time scales, we use the numerical solution of a 3-dimensional reaction diffusion equation with thousands of randomly releasing point sources to model diffusion of calcium in a cardiac cell. We have previously constructed a specialized program to handle the application of spontaneous calcium release in a single cardiac cell [7]. We have shown that the scheme is numerically sound with thorough convergence study [6] and rigorous convergence analysis [14], but the long-time biophysical results in [6] were inappropriate, although the model derivation is reasonable [9]. The key contribution of [6] was to enable reaching large final times by providing an efficient parallel implementation. This brought out the flaws in the values of model parameters that remain hidden in short-term simulations such as in [9].

In this paper, we provide a reduction of the 3-D random system to 1-D deterministic system to find biophysically appropriate regimes in parameter space for long-time behavior. This then informs us of the parameter region to explore in the full 3-D system. Our approach can be applied to other sophisticated problems. The work and reduction technique presented here can be viewed as a rigorous extension of the studies conducted

*Corresponding author. Email: bpeercy@umbc.edu 
in [4] where we performed, in a stochastic setting, an extensive study of the model parameters and showed that wave propagation is sensitive to pump and release strengths. The result of our technique indicates that we can use reduction to get information on this complicated type of problem.

We motivate the 3-D to 1-D reduction with rational biophysical assumptions derived from calcium wave properties and with an asymptotic approximation of the 3-D system which has the 1-D system satisfying a solvability condition. We transform the random release function, which saturates in probability as a Hill function of local calcium concentration, by increasing the Hill coefficient and maximum probability to 1 . We then show that mapping the 1-D model back to the 3-D environment retains the important biophysical parameter regimes even in the random case. With this assessment, we can now characterize biophysically interesting long-time scale behavior such as a sustained traveling wave that was previously unobserved in this model.

Specifically, this paper focuses on critical parameters that have a significant effect on the dynamic behavior of calcium waves in order to obtain experimentally seen longtime scale behaviors. More precisely, we look at the behavior of the model when wave propagation-sensitive terms (pump and release) are varied including values used in the literature and derive a range of parameter values for given behaviors.

The paper is organized as follows. Section 2 describes a 3-D stochastic model of calcium flow in heart cell and introduces its 1-D deterministic equivalent. Section 3 describes the implementation of the 3-D and 1-D models using the finite element method. Section 4 describes 3-D and 1-D model dynamics and characterizations. We finish the paper with a discussion in Section 5 .

\section{Model}

The dynamics of calcium release and calcium uptake are critical in the process of contraction and relaxation of cardiac cells because elevated cytosolic calcium levels are required in the binding of myofilaments and calcium removal is required for relaxing contraction. Under certain conditions, such as high sarcoplasmic reticulum (SR) calcium load [8], spontaneous release from ryanodine receptors can occur and cause subsequent receptors to activate, leading to the initiation and propagation of a calcium wave and a non-uniform cell contraction. Self-initiated intracellular calcium waves have the potential to lead to cardiac arrhythmias (irregular heart beats). Although arrhythmias are mainly attributed to electrical dysfunction, they have been linked, from a sub-cellular point of view, to spontaneous calcium sparks self-organizing into waves [2].

\section{$2.1 \quad 3-D$ model description}

A mathematical model of calcium flow in the heart cell, as presented in [9] is given by the system of coupled, time-dependent reaction diffusion equations

$$
\begin{aligned}
& \frac{\partial c}{\partial t}=\nabla \cdot\left(D_{c} \nabla c\right)+R_{2}+R_{1}-J_{\text {pump }}+J_{\text {leak }}+J_{\mathrm{CRU}} \\
& \frac{\partial f}{\partial t}=\nabla \cdot\left(D_{f} \nabla f\right)+R_{1} \\
& \frac{\partial b}{\partial t}=\nabla \cdot\left(D_{b} \nabla b\right)+R_{2}
\end{aligned}
$$


on a three-dimensional rectangular domain $\Omega$ with no-flux boundary conditions, where $c$ represents the cytosolic calcium ion concentration, $f$ represents the free fluorescent calcium indicator concentration, and $b$ represents the free endogenous calcium buffer concentration. The non-linear reaction terms, $R_{1}$ and $R_{2}$, follow the chemical reactions and are given by

$$
\begin{gathered}
c+f \underset{k_{1}^{-}}{\stackrel{k_{1}^{+}}{\rightleftharpoons} F} \quad \Leftrightarrow \quad R_{1}=-k_{1}^{+} c f+k_{1}^{-} F=-k_{1}^{+} c f+k_{1}^{-}\left(F_{T}-f\right), \\
\underset{\substack{k_{2}^{-} \\
k_{2}^{-}}}{\stackrel{k^{+}}{\rightleftharpoons}} \quad \Leftrightarrow \quad R_{2}=-k_{2}^{+} c b+k_{2}^{-} B=-k_{2}^{+} c b+k_{2}^{-}\left(B_{T}-b\right),
\end{gathered}
$$

where $F$ and $B$ are bound indicator and bound buffer species, respectively. Using conservation of buffers, having assumed equal diffusion of bounded and unbounded buffer, the terms $F$ and $B$ are substituted with $F_{T}-f$ and $B_{T}-b$, where $F_{T}$ and $B_{T}$ are total buffer concentration. The values of constants used in the model (and in [6]) are listed in Table 1.

The pump term in (1) is formulated as

$$
J_{\text {pump }}(c)=V_{\text {pump }} \frac{c^{n_{\text {pump }}}}{K_{\text {pump }}^{n_{\text {pump }}}+c^{n_{\text {pump }}}},
$$

where $V_{\text {pump }}$ is the maximum SR pump rate measured in $\mu \mathrm{M} / \mathrm{s}, K_{\text {pump }}$ is the SR pump sensitivity constant with value of $0.184 \mu \mathrm{M}$, and $n_{\text {pump }}$ is the SR pump Hill coefficient with value of 4 . The term $J_{\text {leak }}$ is taken to be a constant; at rest, the calcium leak balances the pump (i.e., $J_{\text {leak }}=J_{\text {pump }}\left(c_{\text {ini }}\right)$ for $c_{\text {ini }}=0.1 \mu \mathrm{M}$ ). The release of calcium ions through clusters of ryanodine receptors called calcium release units (CRUs) located at discrete locations, $\hat{\mathbf{x}} \in \Omega_{s}$, is modeled with the expression

$$
J_{\mathrm{CRU}}(c, \mathbf{x}, t)=\sum_{\hat{\mathbf{x}} \in \Omega_{s}} \sigma S_{\hat{\mathbf{x}}}(c, t) \delta(\mathbf{x}-\hat{\mathbf{x}}),
$$

where $\sigma$ is the amount of calcium injected per CRU in units of $\mu \mathrm{M} \mu \mathrm{m}^{3} / \mathrm{ms}$. The term $\sigma$ is related to the calcium current from a spark $I_{\mathrm{SR}}$ by $\sigma=I_{\mathrm{SR}} /(2 F)$, with $F=96,485.3 \mathrm{C} / \mathrm{mol}$, the Faraday constant. The Dirac delta distribution $\delta(\mathbf{x}-\hat{\mathbf{x}})$ models the injection of calcium as a point source at each CRU location $\hat{\mathbf{x}} \in \Omega_{s}$. The indicator function $S_{\hat{\mathbf{x}}}(c, t)$ is defined by

$$
S_{\hat{\mathbf{x}}}(c, t)= \begin{cases}1 & \text { if } \alpha \leq J_{\mathrm{prob}}(c), \\ 0 & \text { if } \alpha>J_{\operatorname{prob}}(c),\end{cases}
$$

with probability

$$
J_{\text {prob }}(c)=P_{\max } \frac{c^{n_{\text {prob }}}}{K_{\text {prob }}^{n_{\text {prob }}}+c^{n_{\text {prob }}}},
$$

for $\alpha$ is a random number, between 0 and 1, generated from a uniform random distribution, $P_{\max }$ is the maximum probability rate in $\mathrm{ms}^{-1}$ and $K_{\text {prob }}$ is the probability sensitivity constant with value of $5 \mu \mathrm{M}$. When a CRU opens, that is, $S_{\hat{\mathbf{x}}}(c, t)$ changes 


\begin{tabular}{|c|c|c|}
\hline Parameter & Description & Values/Units \\
\hline$c$ & Calcium concentration & $\mu \mathrm{M}$ \\
\hline$c_{\text {ini }}$ & Initial calcium concentration & $0.1 \mu \mathrm{M}$ \\
\hline$D_{c}$ & Calcium diffusion coefficient & $\begin{array}{l}\operatorname{diag}\left(D_{c_{x}}, D_{c_{y}}, D_{c_{z}}\right) \\
=\operatorname{diag}(150,150,300) \quad \mu \mathrm{m}^{2} / \mathrm{s}\end{array}$ \\
\hline$f$ & Mobile buffer concentration & $\mu \mathrm{M}$ \\
\hline$f_{\text {ini }}$ & Initial mobile buffer concentration & $45.9184 \mu \mathrm{M}$ \\
\hline$F_{T}$ & Total mobile buffer concentration & $50 \mu \mathrm{M}$ \\
\hline$D_{f}$ & Mobile buffer diffusion coefficient & $\begin{array}{l}\operatorname{diag}\left(D_{f_{x}}, D_{f_{y}}, D_{f_{z}}\right) \\
=\operatorname{diag}(10,10,20) \quad \mu \mathrm{m}^{2} / \mathrm{s}\end{array}$ \\
\hline$k_{1}^{+}$ & Forward reaction rate & $80 \mu \mathrm{M}^{-1} \mathrm{~s}^{-1}$ \\
\hline$k_{1}^{-}$ & Backward reaction rate & $90 \mathrm{~s}^{-1}$ \\
\hline$b^{1}$ & Mobile buffer concentration & $\mu \mathrm{M}$ \\
\hline$b_{\text {ini }}$ & Initial mobile buffer concentration & $111.8182 \mu \mathrm{M}$ \\
\hline$B_{T}$ & Total stationary buffer concentration & $123 \mu \mathrm{M}$ \\
\hline$D_{b}$ & Stationary buffer diffusion coefficient & $\operatorname{diag}(0,0,0) \quad \mu \mathrm{m}^{2} / \mathrm{s}$ \\
\hline$k_{2}^{+}$ & Forward reaction rate & $100 \mu \mathrm{M}^{-1} \mathrm{~s}^{-1}$ \\
\hline$k_{2}^{-}$ & Backward reaction rate & $100 \mathrm{~s}^{-1}$ \\
\hline$V_{\text {pump }}$ & Maximum pump strength & $\mu \mathrm{Ms}^{-1}$ \\
\hline$n_{\text {pump }}$ & Pump Hill coefficient & 4 \\
\hline$K_{\text {pump }}$ & pump sensitivity & $0.184 \mu \mathrm{M}$ \\
\hline$J_{\text {leak }}$ & Leak term & $\approx 0.16 \mu \mathrm{Ms}^{-1}$ \\
\hline$\sigma$ & Source flux amplitude & $\mu \mathrm{M} \mu \mathrm{m}^{3} / \mathrm{ms}$ \\
\hline$\Delta x_{s}=\Delta y_{s}$ & CRU spacing in $x$ - and $y$-direction & $0.8 \mu \mathrm{m}$ \\
\hline$\Delta z_{s}$ & CRU spacing in $z$-direction & $2.0 \mu \mathrm{m}$ \\
\hline$P_{\max }$ & Maximum probability rate & $\mathrm{ms}^{-1}$ \\
\hline$\Omega$ & Rectangular domain in $\mu \mathrm{m}$ & $\begin{array}{l}(-l, l) \times(-l, l) \times(-L, L) \\
=(-6.4,6.4) \times(-6.4,6.4) \times(-32,32)\end{array}$ \\
\hline$t_{\text {open }}$ & CRU opening time & $5 \mathrm{~ms}$ \\
\hline$t_{\text {close }}$ & CRU refractory period & $100 \mathrm{~ms}$ \\
\hline$t_{\text {fin }}$ & Simulation duration & $1,000 \mathrm{~ms}$ \\
\hline
\end{tabular}

state from 0 to 1 , a spark or release of calcium ions occurs for $t_{\text {open }}$ ms; the CRU then closes $\left(S_{\hat{\mathbf{x}}}(c, t)=0\right)$ and enters an inhibition or refractory period of $t_{\text {closed }}$ ms during which it cannot open. In our model, $t_{\text {open }}=5 \mathrm{~ms}$ and $t_{\text {closed }}=100 \mathrm{~ms}$.

\subsection{1-D model description}

A search on wave-sensitive parameters in the full 3-D formulation of the model becomes difficult due to probabilistic calcium release and to the computational cost involved with running a long-time simulation [4]. Instead, we consider a 1-D model based on longitudinal direction ( $z$-direction) of the domain on which a parameter search is performed to gain insight into which parameter sets lead the model to display biophysically acceptable behaviors. By biophysically acceptable behaviors, we mean either wave propagation(s) with recovery or no wave propagation; this is as opposed to, for instance, growth of calcium concentration without bound in some simulations in [6].

The motivation to use a 1-D version of the model is based on the fact that in $3-\mathrm{D}$, a wave propagation after self-initiation at a random point in space is nearly a plane wave in the longitudinal direction of the cell. Furthermore, once a wave is initiated, it triggers 
CRUs in the front of the wave to appear deterministic instead of stochastic; this is due to high calcium concentration. Indeed, if we assume that during a wave propagation, the calcium concentration is high compared to $K_{\mathrm{prob}}$, the firing rate probability of a CRU in the neighborhood of the wave front tends to $P_{\max }$ and the probability $P_{n}$ of firing within an interval $t \in[0, n]$ is given by

$$
P_{n}=1-\left(1-P_{\max }\right)^{n}
$$

which is the cumulative distribution for the waiting time probability for a geometric distribution. The above probability is near 1 even for a short interval (e.g., $P_{\max }=0.3$ and $n=10 \mathrm{~ms}$ ). The planar and almost deterministic characteristic of calcium wave propagation has also been observed experimentally [11]. Hence, propagation in 3-D has an almost regular and unidirectional property. We formulate a one-dimensional version of the problem based on the unidimensional aspect of calcium diffusion as follows

$$
\begin{aligned}
c_{t} & =D_{c_{z}} c_{z z}+R_{1}+R_{2}-J_{\text {pump }}+J_{\text {leak }}+J_{\mathrm{CRU}}, \\
f_{t} & =D_{f_{z}} f_{z z}+R_{1}, \\
b_{t} & =D_{b_{z}} b_{z z}+R_{2},
\end{aligned}
$$

where $D_{c_{x}}, D_{f_{x}}$, and $D_{b_{x}}$ are effective diffusion coefficients in the longitudinal direction as shown in Table 1.

We can capture the regular aspect of self-initiating waves by changing the model of CRU firing from being stochastic to deterministic by defining $P_{\max }=1$ and having $n_{\text {prob }} \rightarrow \infty$. We obtain the following formula

$$
J_{\text {prob }}(c)= \begin{cases}0 & \text { if } c<K_{\text {prob }} \\ 1 / 2 & \text { if } c=K_{\text {prob }} \\ 1 & \text { if } c>K_{\text {prob }}\end{cases}
$$

which gives the following expression for $J_{\mathrm{CRU}}$

$$
J_{\mathrm{CRU}}(c, \mathbf{x}, t)= \begin{cases}0 & \text { if } c \leq K_{\mathrm{prob}} \\ \sigma \delta(\mathbf{x}-\hat{\mathbf{x}}) & \text { if } c>K_{\mathrm{prob}}\end{cases}
$$

We further expand the expression for $J_{\mathrm{CRU}}$ to take into account the firing mechanism in our model, i.e., CRUs are considered to be point sources, which when firing, open for a period of $t_{\text {open }}$ and close afterward for a recovery period of $t_{\text {closed }}$. We obtain the following expression

$$
J_{\mathrm{CRU}}(c, \mathbf{x}, t)=\sum \sigma H\left(c-K_{\mathrm{prob}}\right)\left[H\left(t-T^{m}\right)-H\left(t-T^{m}-t_{\mathrm{open}}\right)\right] \delta(\mathbf{x}-\hat{\mathbf{x}}),
$$

where $H(x)$ represents the Heaviside function, $\delta(\mathbf{x}-\hat{\mathbf{x}})$ is the Dirac delta distribution with $\hat{\mathbf{x}}$ representing the CRU coordinates, and $T^{m}$ is the $m^{\text {th }}$ time a particular CRU opens due to calcium concentration crossing threshold levels and is defined by

$$
T^{m}=\inf \left\{t \mid c>K_{\text {prob }}, \frac{\partial c}{\partial t}>0, t \geq T^{m-1}+t_{\text {open }}\right\}, \quad m=0,1, \ldots
$$

The above notation for $T^{m}$ is widely used in the literature when modeling fire-diffusefire type models such as the ones presented in [10] and [3]. With the limiting form of $J_{\text {prob }}$ in (7), the firing process (opening and closing of CRU) does not depend on a 
randomly generated number, but rather on the calcium concentration at release sites. In this deterministic setting, a CRU opens (or fires) either when forced to (when it is manually triggered) or when the calcium concentration at that particular CRU location exceeds the value of $K_{\text {prob}}$.

The connection between 3-D and 1-D model can be made more apparent and mathematically rigorous through a non-dimensionalization and asymptotic expansion of a 3-D buffer-free version of the model. If we consider the three-dimensional bufffer-free diffusion equation

$$
\frac{\partial c}{\partial t}=\nabla \cdot\left(D_{c} \nabla c\right)-J_{\text {pump }}+J_{\text {leak }}+J_{\mathrm{CRU}}
$$

on the domain $\Omega=(-l, l) \times(-l, l) \times(-L, L)$ and introduce the non-dimensional parameters

$$
\hat{c}=c / c_{\text {ini }}, \quad \hat{x}=x / l, \quad \hat{y}=y / l, \quad \hat{z}=z / L, \quad \tau=t D_{c_{z}} / L^{2},
$$

the three-dimensional buffer-free model becomes

$$
c_{\tau}=\frac{L^{2}}{D_{c_{z}}}\left(\frac{D_{c_{x}}}{l^{2}} c_{\hat{x} \hat{x}}+\frac{D_{c_{y}}}{l^{2}} c_{\hat{y} \hat{y}}+\frac{D_{c_{z}}}{L^{2}} c_{\hat{z} \hat{z}}-J_{\text {pump }}+J_{\text {leak }}+J_{\mathrm{CRU}}\right) .
$$

We can now use the elongated geometry with $l<L$ of a typical cardiac cell to define a small parameter $\epsilon$ that incorporates key biophysical features of the model by defining $\epsilon=\frac{D_{c_{z}} l^{2}}{D_{c_{x}} L^{2}}$. Using the fact that $D_{c_{x}}=D_{c_{y}}$, we can rewrite the above equation as

$$
\hat{c}_{\tau}=\hat{c}_{\hat{z} \hat{z}}+\frac{1}{\epsilon}\left(\hat{c}_{\hat{x} \hat{x}}+\hat{c}_{\hat{y} \hat{y}}\right)+\frac{L^{2}}{D_{c_{z}}}\left(-\hat{J}_{\mathrm{pump}}+\hat{J}_{\text {leak }}+\hat{J}_{\mathrm{CRU}}\right)
$$

where

$$
\hat{J}_{\text {pump }}=\frac{J_{\text {pump }}}{c_{\text {ini }}}, \hat{J}_{\text {leak }}=\frac{J_{\text {leak }}}{c_{\text {ini }}}, \text { and } \hat{J}_{\mathrm{CRU}}=\frac{J_{\mathrm{CRU}}}{c_{\text {ini }}} .
$$

We assume that the solution to equation (11) can be expanded asymptotically and written under the form

$$
\hat{c}=c_{0}+\epsilon c_{1}+\epsilon^{2} c_{2}+\cdots,
$$

where $c_{0}, c_{1}, \ldots$ are functions of $\tau, \hat{x}, \hat{y}$, and $\hat{z}$. Substituting the asymptotic expansion of $\hat{c}$ and the following functional Taylor's expansions of the terms

$$
\begin{aligned}
& J_{\text {pump }}=V_{\text {pump }} \frac{c_{0}^{n_{\text {pump }}}}{c_{0}^{n_{\text {pump }}}+K_{\text {pump }}^{n_{\text {pump }}}}+V_{\text {pump }} \frac{n_{\text {pump }}\left(\epsilon c_{1}+\epsilon^{2} c_{2}-c_{0}+\cdots\right) c_{0}^{n_{\text {pump }}-1} K_{\text {pump }}^{n_{\text {pump }}}}{\left(c_{0}^{n_{\text {pump }}}+K_{\text {pump }}^{n_{\text {pump }}}\right)^{2}}+O\left(\epsilon^{2}\right), \\
& J_{\mathrm{CRU}}=\sum \sigma H\left(c_{0}+\epsilon c_{1}+\epsilon^{2} c_{2}-K_{\mathrm{prob}}+\cdots\right) \times \\
& {\left[H\left(L^{2} \tau / D_{c_{x}}-T^{m}\right)-H\left(L^{2} \tau / D_{c_{x}}-T^{m}-t_{\text {open }}\right)\right] \delta(\mathbf{x}-\hat{\mathbf{x}})}
\end{aligned}
$$


in (11), we obtain the ordering equations

$$
\begin{aligned}
& O(1 / \epsilon): \quad c_{0 \hat{x} \hat{x}}+c_{0 \hat{y} \hat{y}}=0
\end{aligned}
$$

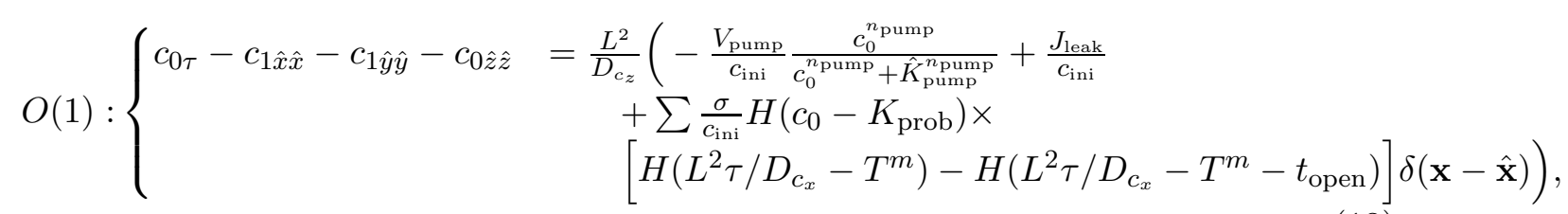

$$
\begin{aligned}
& O(\epsilon):\left\{c_{1 \tau}-c_{2 \hat{x} \hat{x}}-c_{2 \hat{y} \hat{y}}=-\frac{V_{\text {pump }}}{c_{\text {ini }}} \frac{n_{\text {pump }} c_{1} c_{0}^{n_{\text {pump }}-1} K_{\text {pump }}^{n_{\text {pump }}}}{\left(c_{0}^{n_{\text {pump }}}+K_{\text {pump }}^{n_{\text {pump }}}\right)^{2}} .\right.
\end{aligned}
$$

Using separation of variables in Equation (12), we get that

$$
c_{0}(\hat{x}, \hat{y}, \hat{z}, \tau)=h_{0}(\hat{x}, \hat{y}) g_{0}(\hat{z}, \tau)
$$

where $h_{0_{\hat{x} \hat{x}}}+h_{0_{\hat{y} \hat{y}}}=0$. Since $\hat{c}(\hat{x}, \hat{y}, \hat{z}, 0)=\hat{c}_{\text {ini }}$, then $h_{0}(\hat{x}, \hat{y})=K(\hat{z}, \tau)$. From Equation (12), we have that $c_{0}(\hat{x}, \hat{y}, \hat{z}, \tau)=c_{0}(\hat{z}, \tau)$. Let the self-adjoint operator $\mathcal{L}: U \rightarrow U$ be defined as

$$
\mathcal{L}(\phi)=\phi_{\hat{x} \hat{x}}+\phi_{\hat{y} \hat{y}}
$$

where $\phi \in U=H_{0}^{1}(\Omega) \cap L^{2}(\Omega)$ is an adequate function space for our problem (see [14]) on which the standard inner product $\langle f, g\rangle=\int_{\Omega} f g d \Omega$ is used for $f, g \in U$. We can rewrite the first order equation as

$$
O(1): \mathcal{L}\left(c_{1}\right)=\underbrace{c_{0 \tau}-c_{0 \hat{z} \hat{z}}-G}_{F}
$$

with the additional observation that $\mathcal{L}\left(c_{0}\right)=0$; here, $G$ is the right hand side of Equation (13). From the Fredholm alternative theorem, Equation (15) has a solution if $F$ is orthogonal to $c_{0}\left(\left\langle c_{0}, F\right\rangle=0\right)$, or in other words if the equation

$$
\int_{\Omega} c_{0}\left(c_{0 \tau}-c_{0 \hat{z} \hat{z}}-G\right) d \Omega=0
$$

holds, which is equivalent to solving

$$
\int_{\Omega}\left(c_{0 \tau}+c_{0 \hat{z} \hat{z}}-G\right) d \Omega=0
$$

Expanding equation (16) using the first ordering equations, and performing an integration with respect to $\hat{x}$ and $\hat{y}$ over the non-dimensionalized subdomain $[0,1] \times[0,1]$, we obtain 
the $1-\mathrm{D}$ equation

$$
\begin{aligned}
& c_{0 \tau}-c_{0 \hat{z} \hat{z}}-\frac{L^{2}}{D_{c_{z}}}\left(-\frac{V_{\text {pump }}}{c_{\text {ini }}} \frac{c_{0}^{n_{\text {pump }}}}{c_{0}^{n_{\text {pump }}}+K_{\text {pump }}^{n_{\text {pump }}}}+\frac{J_{\text {leak }}}{c_{\text {ini }}}\right. \\
& +N \sum \frac{\sigma}{c_{\mathrm{ini}}} H\left(c_{0}-K_{\mathrm{prob}}\right) \times \\
& \left.\left[H\left(L^{2} \tau / D_{c_{x}}-T^{m}\right)-H\left(L^{2} \tau / D_{c_{x}}-T^{m}-t_{\text {open }}\right)\right] \delta(\hat{z}-\hat{\mathbf{z}})\right)=0,
\end{aligned}
$$

where $N$ is the total number of release units in the $x$ - and $y$-planes. Equation (17) is the buffer-free version $\left(R_{1}=R_{2}=0\right)$ of Equation (6). The addition of buffer species introduces the reaction term $R_{1}+R_{2}$ which acts both as a sink and a source of calcium; our analysis will address this case in Section 4.2.

\section{Implementation}

Our method of model simulation uses the finite element method with linear basis functions. The use of the finite element method and the selection of only linear basis functions in our model simulation is motivated by the presence of the Dirac delta distribution in the term $J_{\mathrm{CRU}}$, since heuristic arguments as well as computational evidence in $[6,7]$ suggest that convergence does hold in the case of linear basis function and no better convergence can be expected from higher-order finite elements. These facts were very recently made rigorous in [14].

\subsection{3-D model implementation}

The 3-D simulations use a special-purpose code for systems of time-dependent reactiondiffusion equations using the finite element method [6]. The method uses implicit timestepping with automatic step size and order control in the state-of-the-art numerical differentiation formulas that are also implemented in the stiff ODE solver ode15s in MATLAB [15]. The Krylov subspace method QMR is used as linear solver kernel inside the Newton method that solves the system of non-linear equations at every time step. The Krylov subspace method allows for a matrix-free form for optimal memory efficiency. The code for the simulation is implemented in $\mathrm{C}$ and parallelized using the MPI standard for communications. See [6] for more details on the numerical method used.

The postprocessing uses MATLAB for visualization. The domain, in units of $\mu \mathrm{m}$, $\Omega=(-6.4,6.4) \times(-6.4,6.4) \times(-32,32)$ contains 6,975 CRUs located at discrete positions in the cell interior. The CRUs have the spacings $\Delta y_{s}=\Delta x_{s}=0.8 \mu \mathrm{m}$ in the $x$ - and $y$-directions and $\Delta z_{s}=2.0 \mu \mathrm{m}$ in the $z$-direction of the cell; no CRU is located on the boundary of the cell. The values of $\Delta x_{s}, \Delta y_{s}$, and $\Delta z_{s}$ are the same CRU spacings as used in [9]. Figure 1 shows the domain $\Omega$ with CRUs located at discrete sites.

All simulations of the 3-D model are run on the 86-node cluster tara in the UMBC High Performance Computing Facility (www. umbc.edu/hpcf). Each node of tara has two quad-core Intel Nehalem X5550 processors (2.66 GHz, $8192 \mathrm{kB}$ cache) and $24 \mathrm{~GB}$ of memory. The nodes are connected for parallel communications by a high-performance quad-data rate InfiniBand interconnect. Most simulations presented in this paper are the results of solving the problem on a numerical mesh consisting of $32 \times 32 \times 128$ elements. Runs using 8 compute nodes, with 8 processes per node for a total of 64 parallel MPI processes, typically take on the order of 20 minutes. Considering that each such run in serial would take on the order of 21 hours, it is clear that only the use of a large 


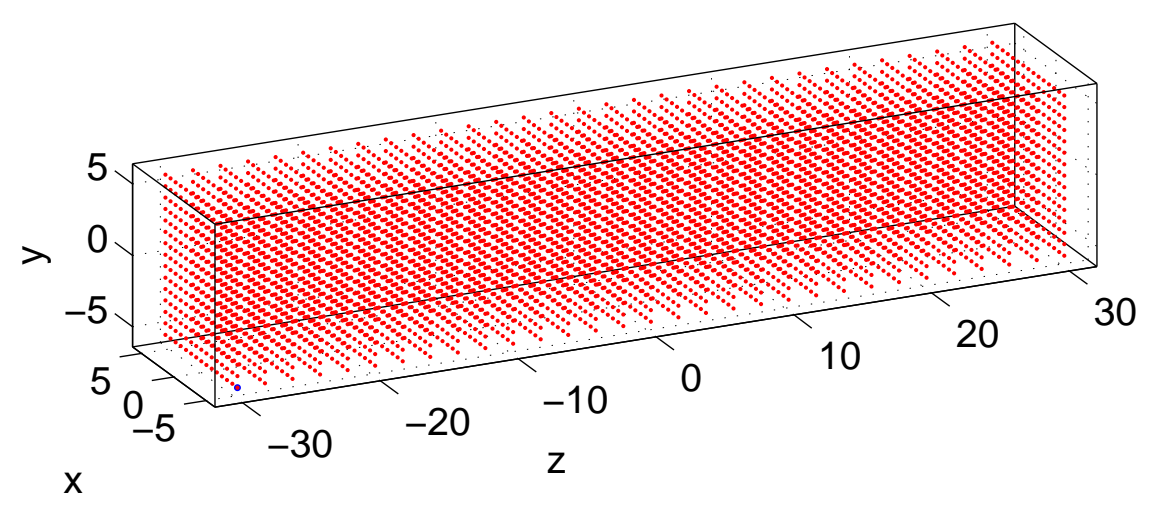

Figure 1. Domain $\Omega$ with all 6,975 CRUs represented as dots.

parallel computer enables production runs of these simulations, in particular since the stochasticity in the model requires repeated simulations for the same parameter values.

\section{$3.2 \quad 1-D$ model implementation}

The 1-D simulations use a 1-D finite element method code implemented in MATLAB; the code makes use of MATLAB's stiff ODE solver ode15s. The mesh resolution used consists of 4 points between CRUs; that is to say, when the number of CRUs in our simulation is $n$ (CRUs are always nodal points), the mesh size consists of $4(n+1)+1$ elements. The distance used between each CRU is $2.0 \mu \mathrm{m}$. Since our 1-D model is deterministic and our purpose is to classify wave behavior (see Section 4), we can use a domain that consists of 3 CRUs and obtain a simulation result over $120 \mathrm{~ms}$. On a standard laptop with a $3.2 \mathrm{GHz}$ processor and $8.0 \mathrm{~GB}$ of memory, such studies take only about 5 minutes, which makes them accessible to parameter studies with large numbers of cases. The 1-D FEM code used in this paper can be obtained through the authors.

\section{Characterization of wave behavior}

\subsection{1-D Characterization}

The deterministic aspect of the one-dimensional model helps in defining conditions that dictate types of behaviors exhibited by the model. Hence, the dynamics fall into one of the following three categories: blow-up, no-wave, and wave. Specifically, a blow-up scenario occurs when an initial forced release of calcium at a particular CRU triggers the opening of its neighboring CRUs; and after the CRU refractory period, further calcium release occurs due to calcium concentration being above threshold level; this dynamic results in an overall calcium concentration growth without bound. A dynamic is classified as a 
no-wave when an initial release of calcium does not result in any triggering of neighboring CRUs; this dynamic results in no wave propagation. A dynamic is classified as a wave when a forced release of calcium at a particular CRU triggers the opening of neighboring CRUs resulting in a wave propagation and after the CRU refractory period, calcium concentration has lowered to basal level. Based on the deterministic aspect of the 1-D model, we can express the above dynamics in terms of CRU opening times. Defining $T_{p}^{m}$ as in Equation (8) to be the $m$ th release occurrence of CRU at position $\hat{x}_{p}$ forced to release at time $T_{p}^{1}$, a no-wave scenario corresponds to the condition

$$
\left\{T_{p}^{m} \mid m \geq 2\right\} \cup\left\{T_{p \pm 1}^{m} \mid m \geq 1\right\}=\emptyset,
$$

where neither a second firing at the $p^{\text {th }}$ CRU nor a firing of an adjacent CRU occurs. A blow-up scenario corresponds to the condition

$$
\text { for all } m \text {, there exists } T_{p}^{m+1} \text { such that } T_{p}^{m+1}>T_{p}^{m} \text {, }
$$

so there will always occur a next firing at the $p^{\text {th }}$ CRU. A wave scenario corresponds to the condition

there exists $T_{p \pm 1}^{1}$ and there does not exist $T_{p \pm 1}^{2}$,

so the neighbors of the $p^{\text {th }}$ CRU will fire exactly once; $T_{p \pm 1}^{m}$ are opening times of neighboring CRUs located at $\hat{x}_{p \pm 1}$. Since the model is deterministic, we restrict $t_{\text {fin }}$ to $120 \mathrm{~ms}$; $t_{\text {fin }}$ defined as such allows the model to capture a CRU releasing calcium, entering refractory period, and potentially re-releasing calcium. Hence, conditions (18), (19), (20) over a period of $120 \mathrm{~ms}$ simplify to there does not exist $T_{p \pm 1}^{1}$; there exists $T_{p}^{2}$; and there exists $T_{p \pm 1}^{1}$, but there does not exist $T_{p \pm 1}^{2}$ respectively. Characterization of wave phenomena is another reason why the use of a lower dimensional and deterministic model is useful in exploring wave-sensitive parameters, as the process of classifying behaviors in the system can be automated.

With the dynamics being well-defined, we explore wave-sensitive parameters ( $V_{\text {pump }}$, and $\sigma$ ) using a method similar to the bisection method; that is to say, for each value of $V_{\text {pump }}$, our algorithm increases or decreases $\sigma$ according to the obtained simulation behavior in order to locate threshold values above and below which the model displays wave, blow-up, and no-wave characteristics.

\section{$4.2 \quad 1-D$ Results}

We have performed a parameter study on the 1-D model (6) without buffer species $\left(R_{1}=R_{2}=0\right)$; Figures 2(a)-(d) show a simulation run resulting in blow-up behavior. In Figure 2(a), a forced spark is initiated for the CRU located at $x=2 \mu \mathrm{m}$; Figure 2(b) shows subsequent CRU firings due to calcium diffusion causing calcium concentration to climb above firing threshold at the neighboring CRUs. In Figure 2(c), no further firing occurs due to CRUs being in their refractory periods $(t \leq 100 \mathrm{~ms}$ ); Figure 2(d) shows more firing occurring due to calcium concentration being above threshold level $(t>105 \mathrm{~ms})$. Running the simulation for a longer period in this scenario will result in the same behavior repeating itself at higher calcium concentration. A wave behavior scenario would be similar to Figures 2(a)-(c); however, at time $t \geq 105 \mathrm{~ms}$ the calcium concentration would be below threshold level and no subsequent firing would occur. In a no-wave scenario, a peak of calcium (spark) as shown in Figure 2(a) would occur, but 


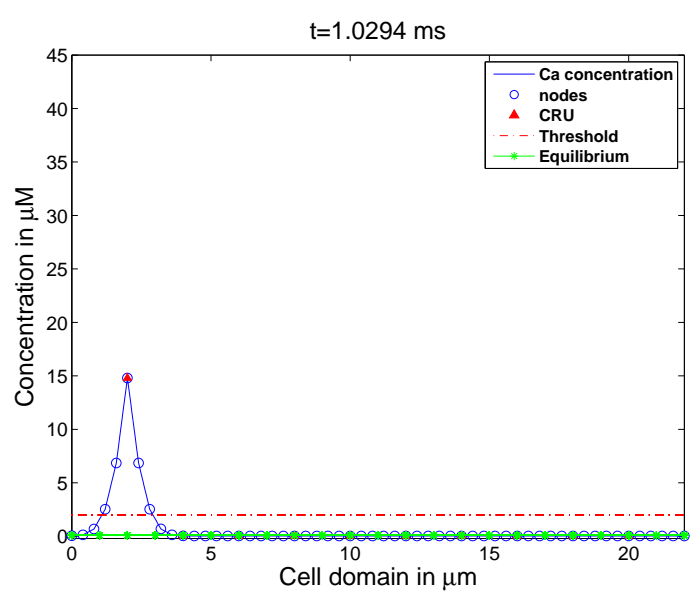

(a)

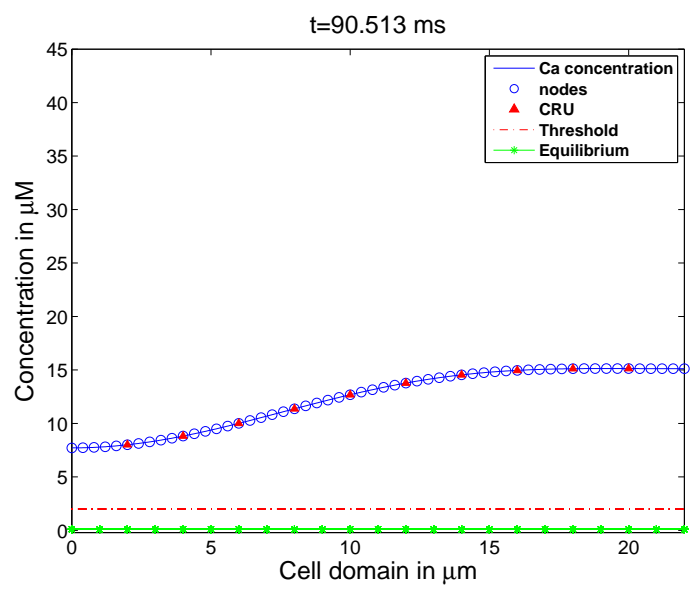

(c)

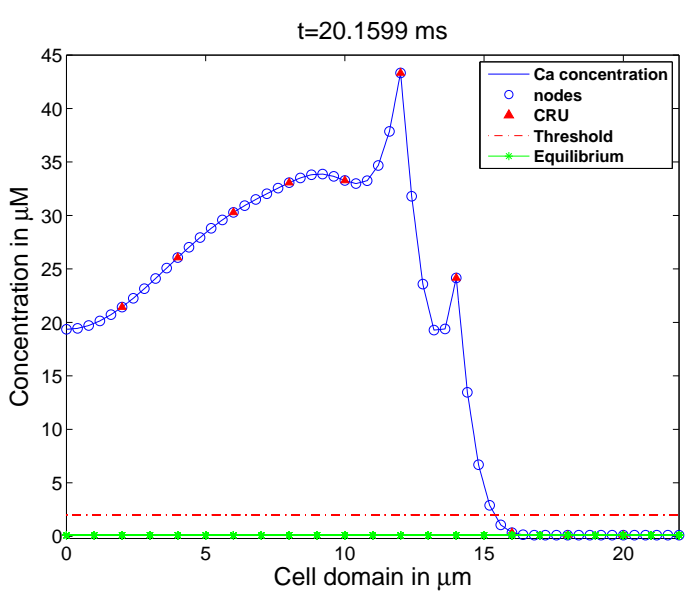

(b)

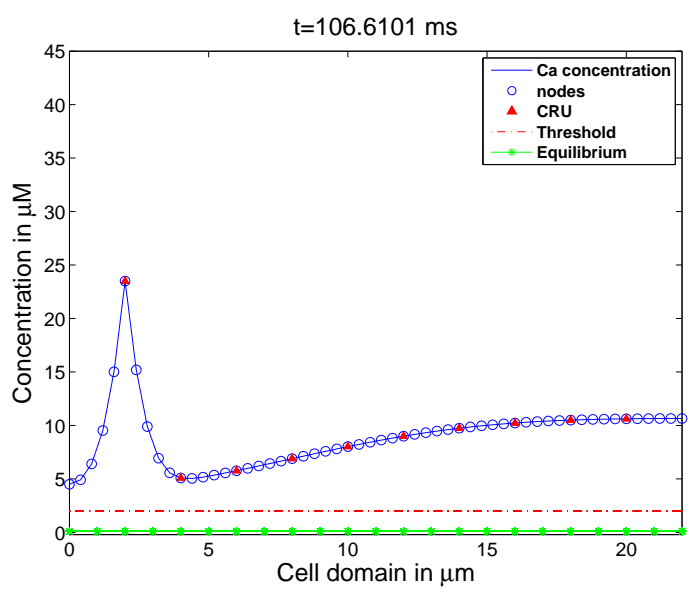

(d)

Figure 2. Instance of a blow-up behavior. (a) Initial condition of simulation: forced activation of CRU at position $x=2 \mu \mathrm{m}$. (b) Wave propagation caused by firing of subsequent CRUs due to calcium concentration crossing threshold. (c) Uptake of calcium; no firing due to CRUs being in resting period. (d) Re-firing of CRU after resting period due to calcium concentration still above threshold level.

propagation would not go as far as the next CRU position resulting in the single forced CRU firing.

The results of our parameter search in the buffer-free version of the model is shown in Figure 3. The graph in Figure 3 shows the model behaviors as a function of $\sigma$ (release strength) and $V_{\text {pump }}$ (pump strength); higher values of $V_{\text {pump }}$ (greater than $0.1 \mu \mathrm{M} / \mathrm{ms}$ ) increases the region of transitional behaviors between the no-wave region located in the lower part region of Figure 3, the wave region (middle part of Figure 3) and the blowup-wave region (upper part of the region Figure 3). More importantly, Figure 3 shows that for $V_{\text {pump }}<0.1 \mu \mathrm{M} / \mathrm{ms}$, only a transition from no-wave behaviors to blow-up-wave behaviors is possible, and no wave behaviors are obtainable. We will refer to the point on Figure 3 where we transition from having two types of wave behaviors to three types as codimension-two point.

The result of including buffer species in the model is summarized in Figure 4. The addition of buffers has the effect of shifting up and to the right the wave regions previously obtained in Figure 3. The shift in the position of the codimension-two point shows that buffers have the intuitive effect of requiring a higher level of release to get a wave propagation. The introduction of buffers in the model also has the effect of increasing 


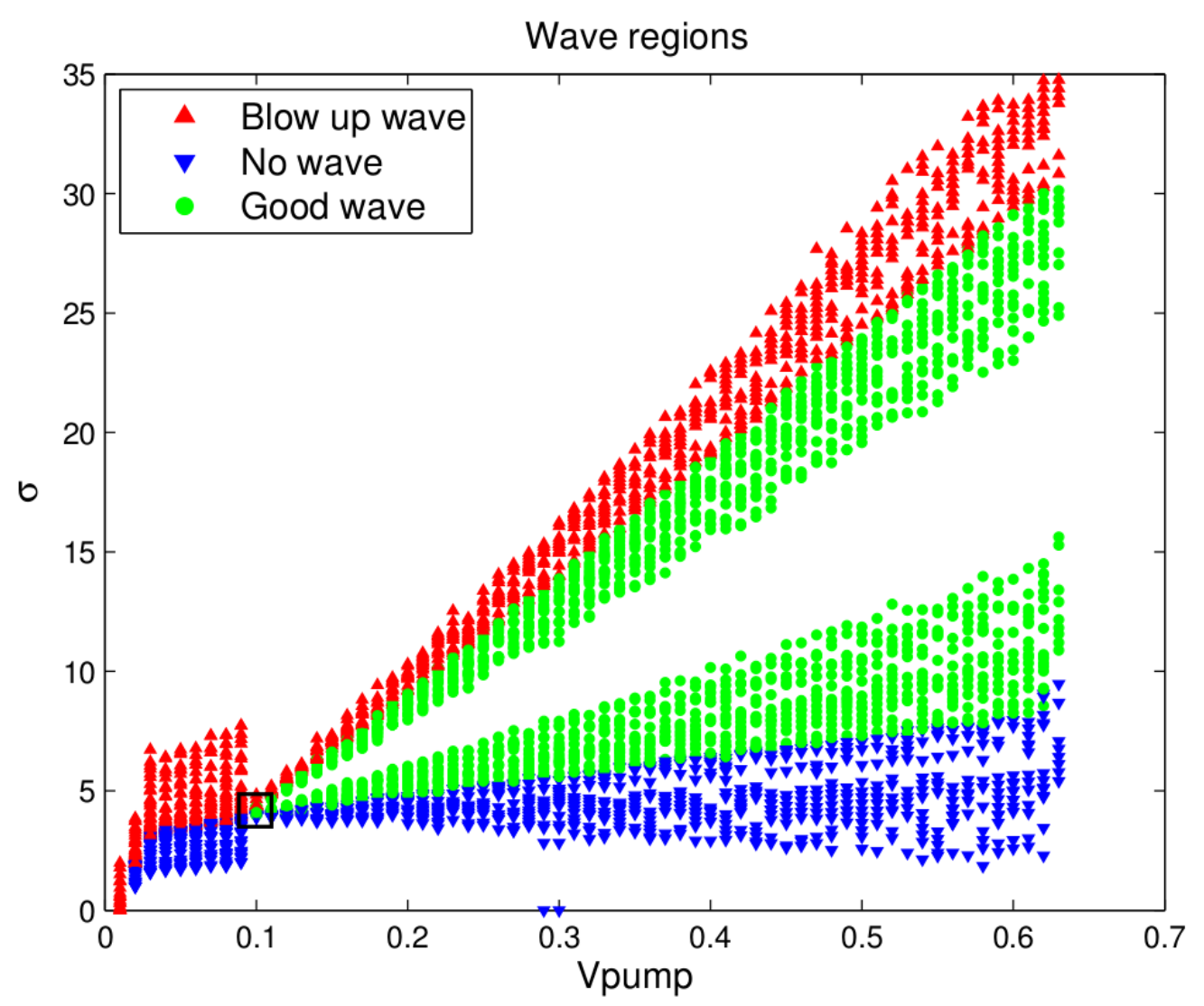

Figure 3. Diagram of Dynamic Behavior for 1-D Model without Buffers. Parameter region for no waves $(\nabla)$ and blow-up waves $(\Delta)$ characterize behavior for pump values below the co-dimesion two point (square outline) beyond which a wave parameter region (o) is apparent contiguously between no waves and blow-up. $V_{\text {pump }}$ is measured in $\mu \mathrm{M} / \mathrm{ms}$ and $\sigma$ is measured in $\mu \mathrm{M} \mu \mathrm{m}^{3} / \mathrm{ms}$.

the range of $V_{\text {pump }}$ and $\sigma$ for which there is no wave propagation with recovery.

The shift in $V_{\text {pump }}$ and $\sigma$ can be explained through the expression of the reaction term $R_{1}+R_{2}$. Indeed the term $k_{1}^{+} c f+k_{2}^{+} c b$ acts as a sink of calcium and is on the order of $100 \mu \mathrm{M} / \mathrm{ms}$ around CRUs, while the term $k_{1}^{-}\left(F_{T}-f\right)+k_{2}^{-}\left(B_{T}-b\right)$ acts as a source of calcium is on the order of $10 \mu \mathrm{M} / \mathrm{ms}$. If we consider the critical values of the codimensiontwo point in the plane, the values of the reaction term acting as sink and source explain both the direction and magnitude of shift from the unbuffered $\left(V_{\text {pump }} \approx 0.1 \mu \mathrm{M} / \mathrm{ms}\right.$ and $\sigma \approx 2 \mu \mathrm{M} / \mathrm{ms}$ ) to the buffered model $\left(V_{\text {pump }} \approx 5 \mu \mathrm{M} / \mathrm{ms}\right.$ and $\sigma \approx 170 \mu \mathrm{M} / \mathrm{ms}$ ).

\subsection{3-D Characterization}

In 3 -D, our method of characterizing simulation runs is done using computational line scans produced from integrating calcium concentration along a specific plane running in the longitudinal axis of the 3-D domain. Line scans are experimentally used visualization tools that summarize in a single figure the overall behavior of the simulation. Examples of computational line scans can be seen in Figure 5; the horizontal axis corresponds to time (from $0 \mathrm{~ms}$ to $1000 \mathrm{~ms}$ ), while the vertical axis corresponds to the longitudinal axis taken from the cell domain. Each computational line scan plots calcium concentration (using a colorbar) at various times of the simulation along points of the longitudinal axis. Mathematically, a computational line scan corresponds to the surface plot of $c_{L}(y, z, t)$ 


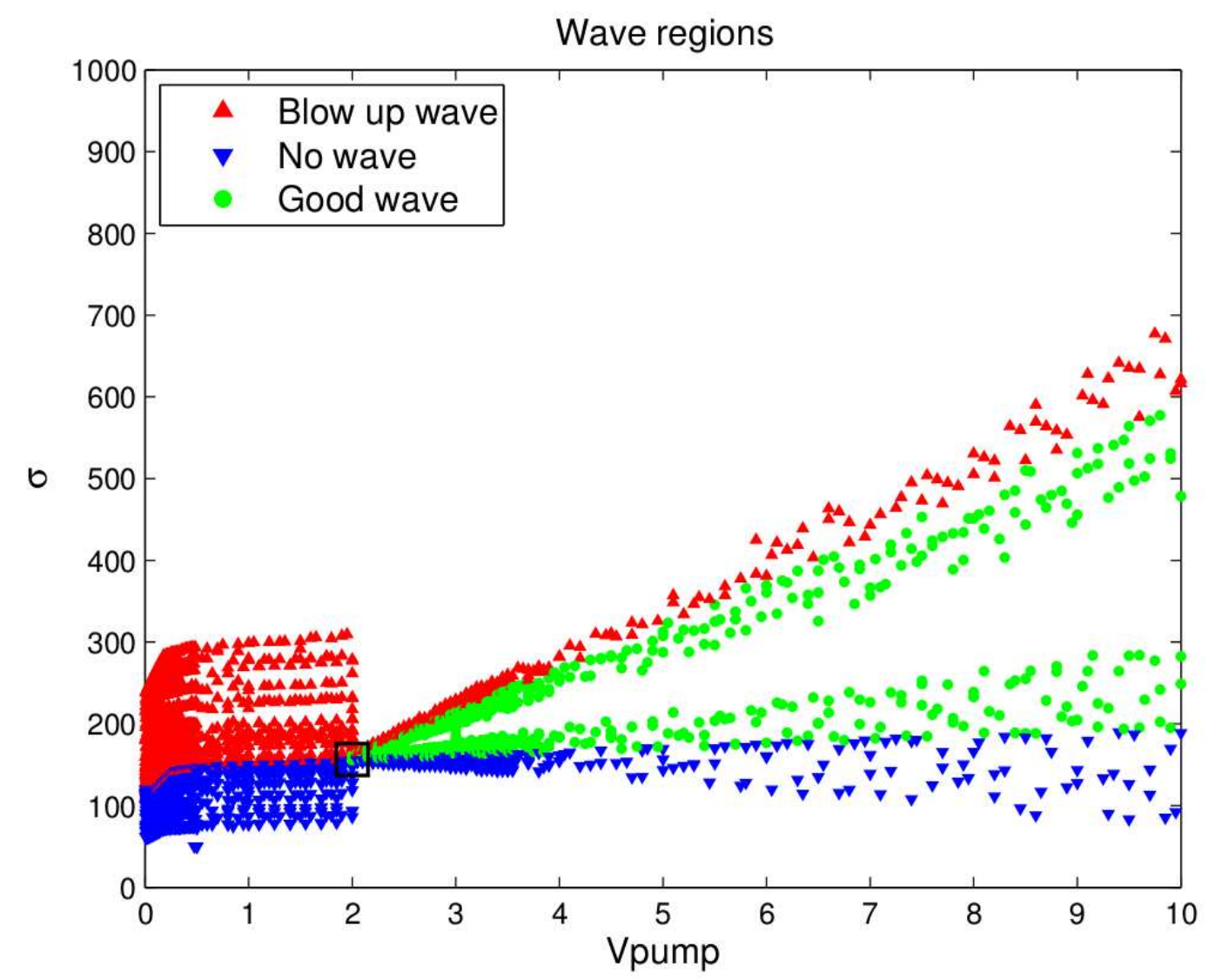

Figure 4. Diagram of Dynamic Behavior for 1-D Model with Buffers. Parameter region for no waves $(\nabla)$ and blow-up waves $(\Delta)$ characterize behavior for pump values below the co-dimesion two point (square outline) beyond which a wave parameter region ( $\circ$ ) is apparent contiguously between no waves and blow-up. However, the codimension two point has shifted in $V_{\text {pump }}$ and $\sigma$ compared to Fig 3. $V_{\text {pump }}$ is measured in $\mu \mathrm{M} / \mathrm{ms}$ and $\sigma$ is measured in $\mu \mathrm{M} \mu \mathrm{m}^{3} / \mathrm{ms}$.

at a particular $y=y_{L}$, with $c_{L}(y, z, t)$ defined as:

$$
c_{L}(y, z, t)=\frac{1}{12.8} \int_{-6.4}^{6.4} c(x, y, z, t) d x
$$

where $c(x, y, z, t)$ corresponds to the calcium component of the solution of the 3-D model. We now use stochastic calcium release and do not force release at any given CRU. In the three-dimensional case, a run is classified as blow-up if the resulting computational line scan shows waves peaking at increasing values and/or no clear recovery can be seen as shown in Figures 5(a) and 5(b); a run is classified as no-wave if the overall calcium concentration stays near basal level almost everywhere as shown in Figures 5(c) and 5(d); and a run is classified as wave when it is neither blow-up nor no-wave as shown in Figures 5(e) and 5(f). We express the above behavior descriptions mathematically. Let $P_{i, m}$ be the local maximum occurring at time $t_{i}$ of $c_{L}\left(y_{L}, z_{m}, t\right)$ the computational line scan at the plane $y=y_{L}$ and at points $z=z_{m}, m=1,2, \ldots$ be defined for $i=1,2, \ldots$ as

$$
P_{i, m}=\max _{t \in\left[0, t_{\text {fin }}\right]}\left\{c_{L}\left(y_{L}, z_{m}, t\right) \mid c_{L}>c_{\text {ini }},\left(t_{i}-t_{i-1}\right) \geq t_{\text {closed }} \text { if } t_{i-1} \text { exists }\right\}
$$


A blow-up scenario corresponds to the condition

$$
\text { for all } m, P_{i+1, m}>P_{i, m} \text {; }
$$

the above condition is based on the fact that if a blow-up occurs, peaks defined as in Equation (22) will be monotonic increasing and will occur at all points $z_{m}$. A no-wave scenario corresponds to the condition

$$
\text { there exists } m \text {, such that } P_{i, m} \text { does not exist; }
$$

the above condition is satisfied when there are points $z_{m}$ at which $c_{L}\left(y_{L}, z_{m}, t\right)$ is still at basal level $c_{\text {ini }}$ or in other words, sparks occur but do not organize into waves. A wave occurs when neither blow-up conditions (23) nor no-wave conditions (24) have been satisfied. Note that in the 3-D study, we do not force any spark to occur; the large number of CRUs $(6,975)$ along with the non-uniform Poisson process governing their firing (see Equations (5) and (4)) is sufficient to determine that within our simulation runtime, a spark will occur with probability close to 1 . Furthermore, to account for the stochastic aspect of the 3-D model, for each set of parameters $V_{\text {pump }}$ and $\sigma$, we run the simulation 5 times and confirm that the appropriate classification is always consistent.

\section{$4.4 \quad 3-D$ results}

The 1-D parameter study shows that the region of wave propagations with recovery (wave region) broadens for large values of $V_{\text {pump }}$ and $\sigma$. Previous simulation work ([6] and [4]) shows behaviors that can be categorized as no-wave and blow-up. The 1-D results presented in Section 4.2 suggests that the previous 3-D simulations may have been restricted to the region in parameter space where only no-wave behaviors or blowup behaviors are observed.

The result of the parameter search in 3-D is displayed in Figure 6. Similar to the 1-D results (shown in Figure 4), there exists a region within which wave propagation with recovery does not occur $\left(0 \mu \mathrm{M} / \mathrm{ms} \leq V_{\text {pump }}<4 \mu \mathrm{M} / \mathrm{ms}\right)$, but only no-wave and blowup behaviors occur. From the results obtained in Figure 6, we see that the wave region appears and broadens as $V_{\text {pump }}$ increases above $4 \mu \mathrm{M} / \mathrm{ms}$. More importantly, there exists a region within which the model displays waves with recovery previously unobserved in the 3-D model. An example of a wave with recovery (also seen experimentally) is shown in Figure 7. This figure displays average calcium concentration along a longitudinal plane (this would be the equivalent to experimental confocal images) at various points of time in the simulation. We can see in Figures 7(a) and 7(b) the occurrence of few sparks (upper left corner near $x=5, z=-30$ ) later on organizing into a wave which then propagates downward right through the cell; Figure 7(c) shows sparks on the middle right side of the cell (near $z=10$ ) organizing into a second wave. In Figures $7(\mathrm{~d})$ and $7(\mathrm{e})$, we see the first and second wave colliding; Figure 7 (f) shows a recovery of calcium concentration. The dynamics shown in Figure 7 is an example of a sustainable traveling wave with recovery. Figure 8 shows isosurface plots of the calcium concentration throughout the three-dimensional cell domain with a critical isolevel of $65 \mu \mathrm{M}$; the processed data is from the same simulation that produced Figure 7, the computational line scan of which is shown in Figure 5(f). 


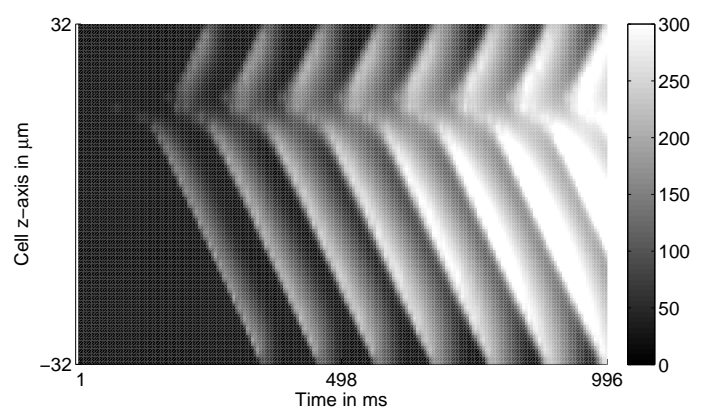

(a) $V_{\text {pump }}=3 \mu \mathrm{Ms}^{-1}, \sigma=100 \mu \mathrm{mol} \mathrm{s}^{-1}$.

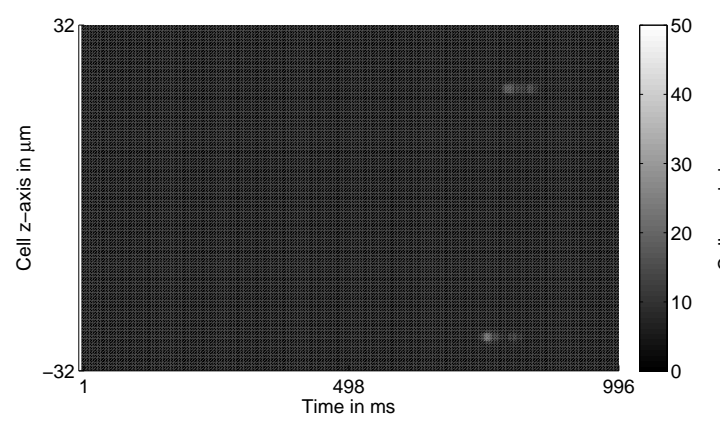

(c) $V_{\mathrm{pump}}=4 \mu \mathrm{Ms}^{-1}, \sigma=100 \mu \mathrm{mol} \mathrm{s}{ }^{-1}$.

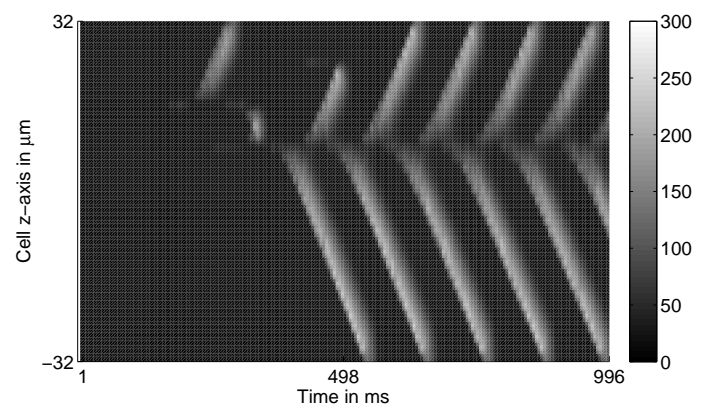

(e) $V_{\text {pump }}=6 \mu \mathrm{Ms}^{-1}, \sigma=130 \mu \mathrm{mol} \mathrm{s}{ }^{-1}$.

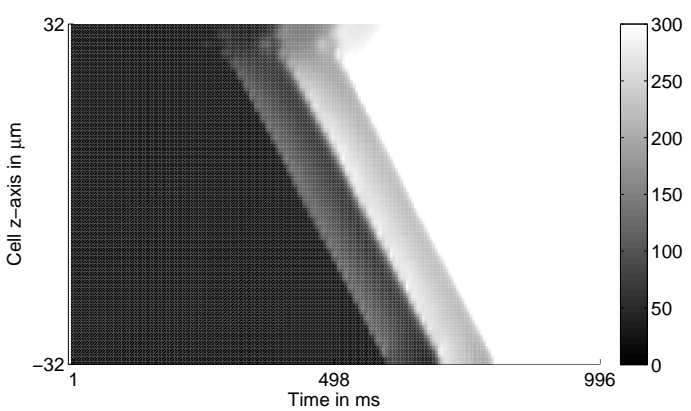

(b) $V_{\text {pump }}=1 \mu \mathrm{Ms}^{-1}, \sigma=80 \mu \mathrm{mol} \mathrm{s}^{-1}$.

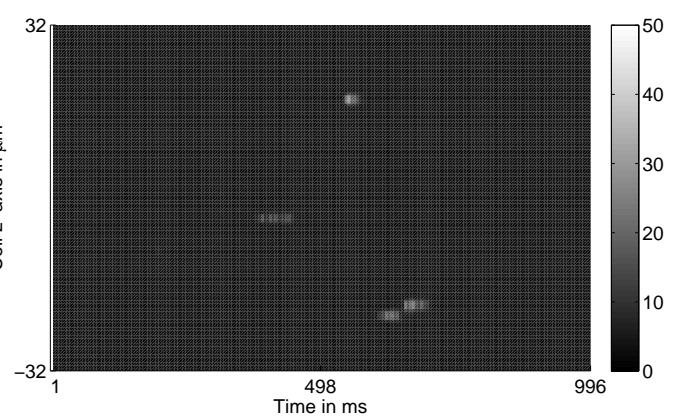

(d) $V_{\text {pump }}=4 \mu \mathrm{Ms}^{-1}, \sigma=100 \mu \mathrm{mol} \mathrm{s}^{-1}$.

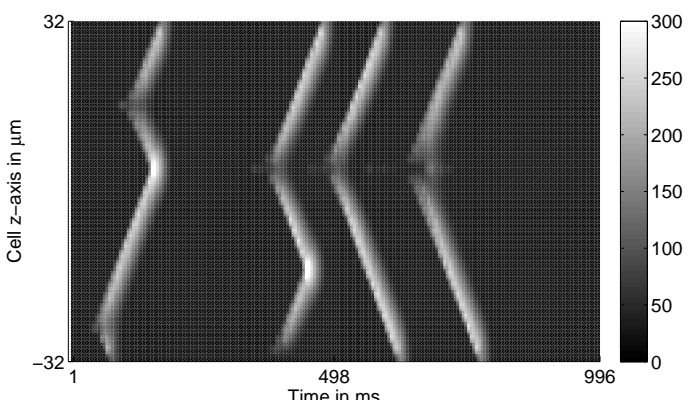

(f) $V_{\text {pump }}=8 \mu \mathrm{Ms}^{-1}, \sigma=150 \mu \mathrm{mol} \mathrm{s}^{-1}$.

Figure 5. Computational line scans showing calcium concentration. (a) and (b) show computational line scans of simulations resulting in a blow-up dynamics; no clear recovery can be seen over the duration of the simulation. (c) and (d) show computational line scans of simulations resulting in a no-wave dynamics; few sparks are observed. (e) and (f) show computational line scans of simulations resulting in a wave dynamics; multiple waves and recovery can be observed over the $1,000 \mathrm{~ms}$ simulations pan. Computational line scans show calcium concentration. 


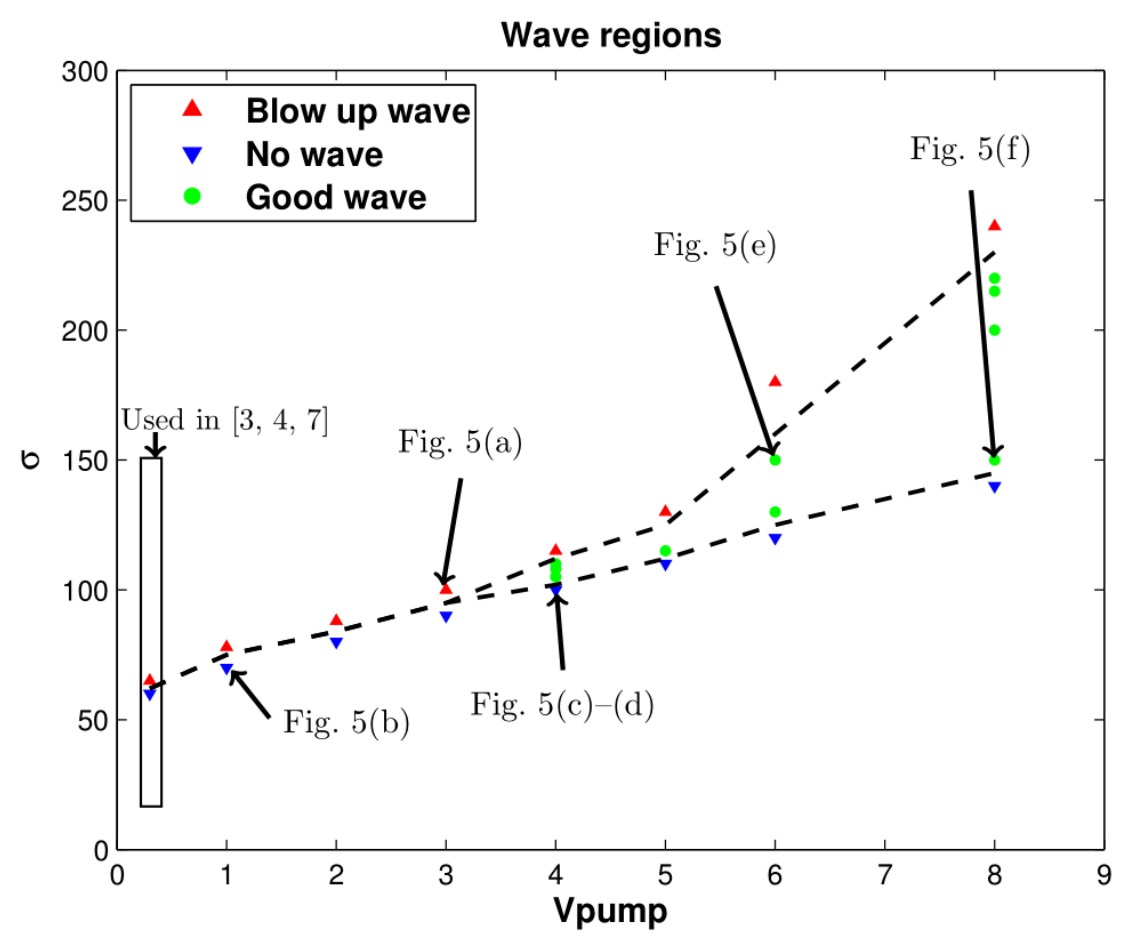

Figure 6. Wave region obtained from parameter study in the three-dimensional stochastic model with buffers. The rectangular box shows the parameter region used in $[4,6,9]$. $V_{\text {pump }}$ is measured in $\mu \mathrm{M} / \mathrm{ms}$ and $\sigma$ is measured in $\mu \mathrm{M} \mu \mathrm{m}^{3} / \mathrm{ms}$.

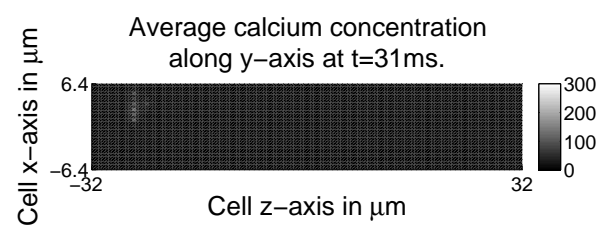

(a)

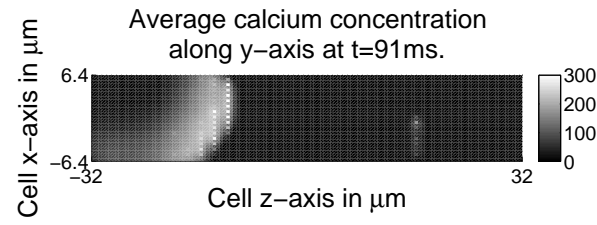

(c)

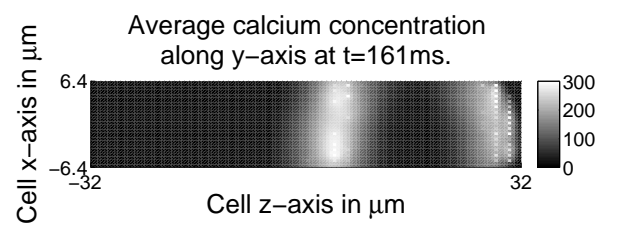

(e)

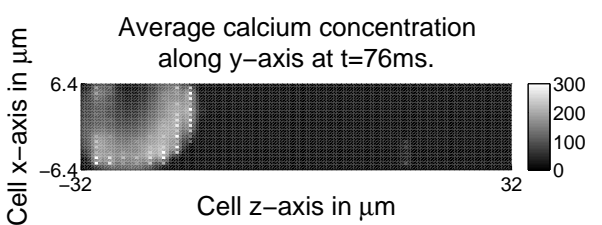

(b)

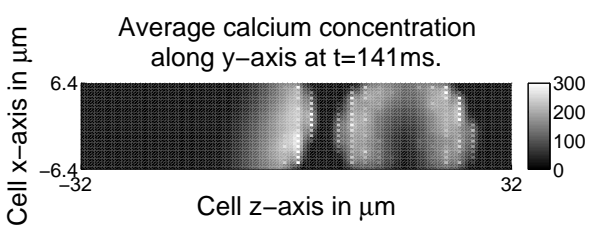

(d)

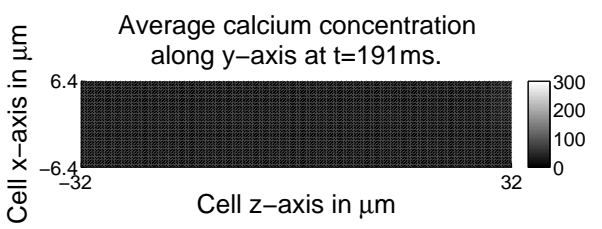

(f)

Figure 7. Confocal images obtained from a simulation run resulting in a wave propagation with recovery (see Figure 5(f)). (a) shows calcium sparks on the upper left corner of the cell. (b) shows sparks organizing into a wave. (c) shows wave propagation (followed by recovery); a few calcium occur on the right side of the cell. (d) shows a second wave originating from the left side of cell. (e) shows first and second wave colliding. (f) shows recovery. $V_{\text {pump }}=8 \mu \mathrm{M} / \mathrm{ms}$ and $\sigma=150 \mu \mathrm{M} \mu \mathrm{m}^{3} / \mathrm{ms}$. 


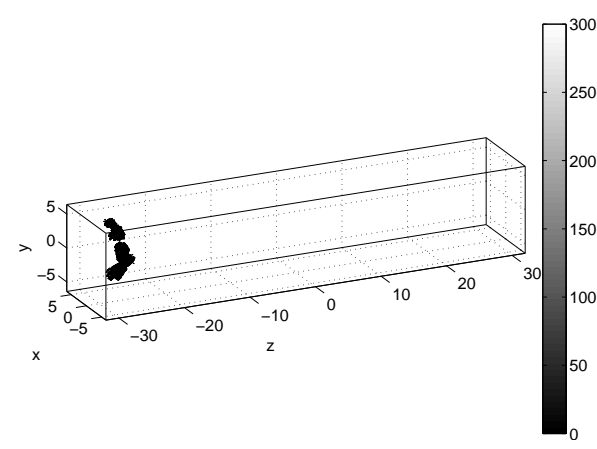

(a)

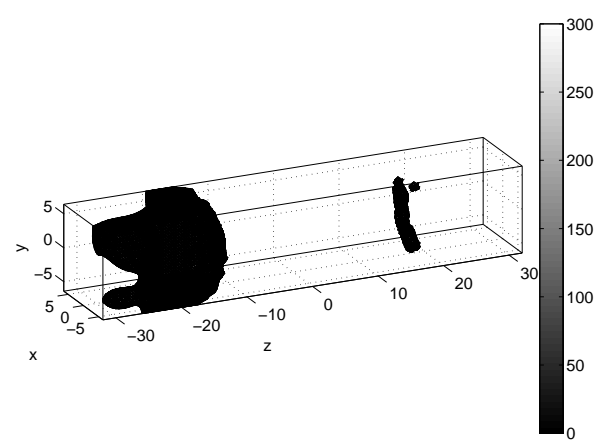

(c)

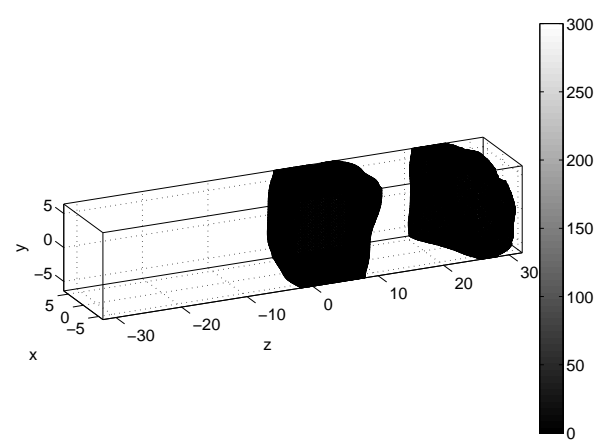

(e)

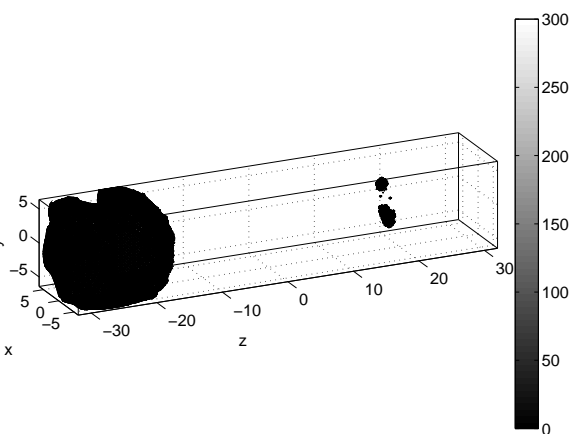

(b)

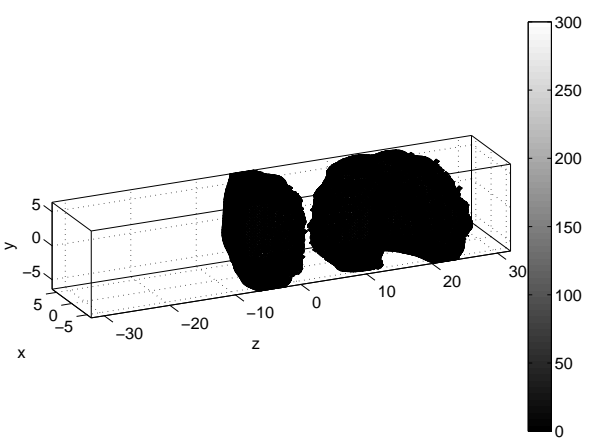

(d)

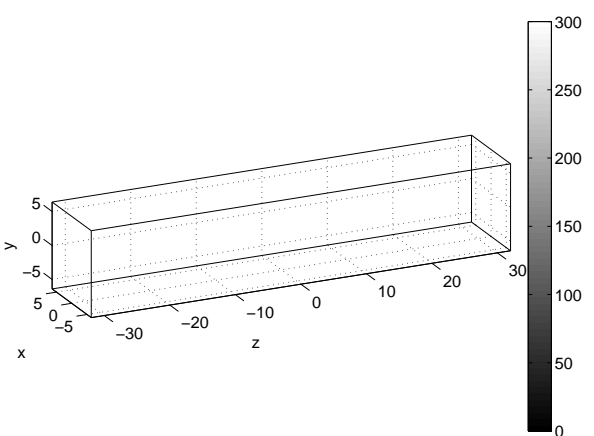

(f)

Figure 8. Isosurface plots of the calcium concentration throughout the three-dimensional cell domain with critical isolevel of $65 \mu \mathrm{M}$. Wave propagation with recovery occurs. (a) shows calcium at basal level. (a) and (b) show calcium sparks. (d) sparks organize into waves. (e) and (f) show propagation of the wave (followed by recovery) towards the right end of the cell. $V_{\text {pump }}=8 \mu \mathrm{M} / \mathrm{ms}$ and $\sigma=150 \mu \mathrm{M} \mu \mathrm{m}^{3} / \mathrm{ms}$. 


\section{Discussion}

We have shown that system features, such as elongated geometry, can generate a small parameter that we can exploit to reduce the dimension of the system. Coupling this with turning calcium release from having a stochastic threshold to having a deterministic one further simplifies a complex system. Then, rather than a 3-D system that requires hours to simulate multiple trials for long enough times, we are able to guide behavior prediction with a 1-D deterministic system within minutes. This template for parameter location from the 1-D system then informs the focused search in 3-D leading to detection of critical parameters and their boundaries. We see this as one viable strategy to reduce complicated systems and yet guide discovery in the full system.

We can provide a concrete example for the dramatic advantage of this approach: Figures 3 and 4, using 1-D simulations, were used to narrow the range of parameter values in the 3-D simulations for Figure 6. Figures 3 and 4 contain results from hundreds of simulation runs, each taking typically about 5 minutes (as described in Section 3.2), thus an exhaustive study of the entire parameter region and most importantly the boundaries between qualitatively different behaviors is computationally feasible. The 3 -D simulations underlying Figure 6 by contrast take 21 hours each in serial or still on the order of 20 minutes even when using 8 compute nodes with two quad-core processors in a parallel cluster (as described in Section 3.1), so it is vital to have a reliable plot for this figure with as few simulations as possible. In the case of Figure 6, only two dozens simulations were needed to capture the bounds of qualitatively different behavior, thus the figure could be feasibly obtained.

Additionally, the study presented in this paper suggests that a wave initiation, propagation, and recovery is likely to occur for release strength larger than $100 \mu \mathrm{M} \mu \mathrm{m}^{3} / \mathrm{ms}$ (corresponding to a CRU current larger than $20 \mathrm{pA}$ ). This result supports the claim of Izu in [8], suggesting that calcium sparks originate from multiple ryanodine receptor currents (more than 30) of smaller intensity. Additionally, the large current has to be coupled with a large pump strength (larger than $4 \mu \mathrm{M} / \mathrm{ms}$ ). This value is on the order of 10 times larger than the one measured experimentally and published in [1]. Increasing the buffers' sensitivity reduces the necessary pump rate to achieve recurrent calcium waves, although not by an order of magnitude. However, other, de facto, calcium stores such as mitochondria or nuclei could serve to reduce the necessary pump strength. Also the rate of the SERCA pump is also sensitive to phospholamban and its inactivation by $\beta$-adrenergic stimulation [12]. This reinforces the necessity to unfold the dynamics in this parameter.

Furthermore, we do not account for emptying of SR stores of calcium which may reduce the recurrence if not the initiation of spontaneous calcium waves, but $\sigma$ can be interpreted as combination of release amplitude and driving force from the calcium store. Thus, for a sufficiently high effective pump rate and a $\sigma$ value that does not support waves, stores can be over filled either experimentally or pathologically driving $\sigma$ higher and perhaps into a wave initiation regime of parameter space. On occasion, initiated waves may not be recurrent without refilling a depleted SR. Our Figure 4 describes this situation if we rewrite $\sigma$ as $\sigma=-g\left(C-C_{S R}\right) \approx g C_{S R}$ splitting the spark current into a conductance and SR dependent driving force for calcium release. Therefore, if store calcium is lost during a normal regular release, an overfull store with $\sigma$ in the wave or even blow-up region may fall below the wave/no-wave threshold for a given $V_{\text {pump }}$, restricting further release prior to re(over)filling the store.

And importantly, while the 1-D buffered system qualitatively and even quantitatively indicates parameter regions of certain dynamic behavior, the 1-D analysis cannot completely capture fundamentally higher dimensional behavior. For example, at the lower 
boundary between waves being and not being generated, in Figure 6, there is greater complexity where we see the emergence of spiral or scroll waves that appear with parameterdependent probability. But the 1-D analysis does suggest where transitions, and therefore sites of potentially interesting dynamics, may be found.

In summary, the one-dimensional leading to the three-dimensional parameter searches presented in this paper have given a map that can be used to pick pump and release values in order to obtain biophysical behaviors. First, we see that there are cases when pump strength dominates the release strength and no propagation occurs (this is still a valid biophysical behavior). Second, we see that there exist combinations of pump strength and release strength for which a non-biophysical behavior can occur; when this combination is picked, simulation, though resulting in wave propagation, sees calcium concentration increasing without bound. Third, we see that there exist combinations of pump and release strength for which wave propagation with recovery occurs (behavior seen experimentally). Furthermore, the 1-D wave region presented in Figure 4 is a good map to the 3-D wave region shown in Figure 6 in the sense that it captures the global characteristics of the $3-\mathrm{D}$ wave region. 


\section{Acknowledgments}

This work was supported by the IQB grant number T36GM078008.

The hardware used in the computational studies is part of the UMBC High Performance Computing Facility (HPCF). The facility is supported by the U.S. National Science Foundation through the MRI program (grant nos. CNS-0821258 and CNS1228778) and the SCREMS program (grant no. DMS-0821311), with additional substantial support from the University of Maryland, Baltimore County (UMBC). See www. umbc.edu/hpcf for more information on HPCF and the projects using its resources.

We would also like to thank to Dr. Ken Spitzer for providing us with experimental data.

\section{References}

[1] C.W. Balke, T.M. Egan, and W.G. Wier, Processes that remove calcium from the cytoplasm during excitation-contraction coupling in intact rat heart cells, Journal of Physiology 474 (1994), pp. 297-316, Available at http://jp.physoc.org/content/474/3/447.abstract?sid= $9742 \mathrm{~d} 35 \mathrm{e}-\mathrm{ff} 82-4 \mathrm{e} 9 \mathrm{~d}-\mathrm{b} 2 \mathrm{bf}-\mathrm{c} 1 \mathrm{f} 20 \mathrm{~d} 10 \mathrm{~d} 56 \mathrm{~d}$.

[2] H. Cheng and W.J. Lederer, Calcium sparks, Physiol. Rev. 88 (2008), pp. 1491-1545, Available at http://physrev.physiology.org/cgi/content/abstract/88/4/1491.

[3] S. Coombes, R. Hinch, and Y. Timofeeva, Receptors, sparks and waves in a fire-diffusefire framework for calcium release, Progress in Biophysics and Molecular Biology 85 (2004), pp. 197-216, Available at http://www.sciencedirect.com/science/article/B6TBN-4C0051B-1/ 2/dba0035d33d48989032da378ce1e4b8e.

[4] Z. Coulibaly, M. Muscedere, M.K. Gobbert, and B.E. Peercy, Long-time simulation of calcium waves in a heart cell to study the effects of calcium release flux density and of coefficients in the pump and leak mechanisms on self-organizing wave behavior, Tech. Rep. HPCF-2009-6, UMBC High Performance Computing Facility, University of Maryland, Baltimore County, 2009, Available at www. umbc. edu/hpcf.

[5] G. Dupont and A. Goldbeter, Properties of intracellular $\mathrm{ca}^{2+}$ waves generated by a model based on $\mathrm{ca}^{2+}$-induced $\mathrm{ca}^{2+}$ release, Biophys. J. 67 (1994), pp. 2191-2204.

[6] M.K. Gobbert, Long-time simulations on high resolution meshes to model calcium waves in a heart cell, SIAM J. Sci. Comput. 30 (2008), pp. 2922-2947.

[7] A.L. Hanhart, M.K. Gobbert, and L.T. Izu, A memory-efficient finite element method for systems of reaction-diffusion equations with non-smooth forcing, J. Comput. Appl. Math. 169 (2004), pp. 431-458.

[8] L.T. Izu, J.R.H. Mauban, C.W. Balke, and W.G. Wier, Large currents generate cardiac Ca $^{2+}$ sparks, Biophys. J. 80 (2001), pp. 88-102.

[9] L.T. Izu, S.A. Means, J.N. Shadid, Y. Chen-Izu, and C.W. Balke, Interplay of ryanodine receptor distribution and calcium dynamics, Biophys. J. 91 (2006), pp. 95-112.

[10] J. Keizer and G.D. Smith, Spark-to-wave transition: saltatory transmission of calcium waves in cardiac myocytes, Biophys. Chem. 72 (1998), pp. 87-100.

[11] P. Lipp and E. Niggli, Microscopic spiral waves reveal positive feedback in subcellular calcium signaling, Biophys. J. 65 (1993), pp. 2272-2276.

[12] D.H. MacLennan and E.G. Kranias, Phospholamban: a crucial regulator of cardiac contractility, Nat. Rev. Mol. Cell Biol. 4 (2003), pp. 566-577, Available at http://dx.doi.org/10.1038/nrm1151.

[13] M. Nivala, C.Y. Ko, M. Nivala, J.N. Weiss, and Z. Qu, Criticality in intracellular calcium signaling in cardiac myocytes, Biophys. J. 102 (2012), pp. 2433-2442.

[14] T.I. Seidman, M.K. Gobbert, D.W. Trott, and M. Kružík, Finite element approximation for timedependent diffusion with measure-valued source, Numer. Math. 122 (2012), pp. 709-723.

[15] L.F. Shampine and M.W. Reichelt, The MATLAB ODE suite, SIAM J. Sci. Comput. 18 (1997), pp. $1-22$. 